\title{
Comparative study of biological characteristics of mesenchymal stem cells isolated from mouse bone marrow and peripheral blood
}

\author{
AHMED LOTFY $^{1}$, YASSER M. EL-SHERBINY ${ }^{2-4}$, RICHARD CUTHBERT ${ }^{2}$, \\ ELENA JONES $^{2}$ and AHMED BADAWY ${ }^{5}$
}

\begin{abstract}
${ }^{1}$ Biotechnology and Life Sciences Department, Faculty of Postgraduate Studies for Advanced Sciences, Beni-Suef University, Beni-Suef 62511, Egypt; ${ }^{2}$ Leeds Institute of Rheumatic and Musculoskeletal Medicine, University of Leeds, Leeds LS9 7TF, UK; ${ }^{3}$ Clinical Pathology Department, Faculty of Medicine, Mansoura University, Mansoura 35516, Egypt; ${ }^{4}$ Department of Biosciences, School of Science and Technology, Nottingham Trent University, Nottingham NG11 8NS, UK; ${ }^{5}$ Medical Experimental Research Center, Faculty of Medicine, Mansoura University, Mansoura 35516, Egypt
\end{abstract}

Received April 10, 2019; Accepted June 14, 2019

DOI: $10.3892 / b r .2019 .1236$

\begin{abstract}
Mesenchymal stromal cells (MSCs) possess self-renewal and multilineage differentiation potential, indicating their prospects as cellular therapeutic agents for regenerative medicine. Although adult bone marrow (BM) is the major source of these cells for clinical use, harvesting requires invasive procedures. Therefore, alternative sources, such as peripheral blood (PB), are needed. The objective of the current study was to compare PB-MSCs and BM-MSCs with regard to their biological characteristics. PB-MSCs and BM-MSCs were isolated from 4-week-old $\mathrm{BALB} / \mathrm{c}$ white mice by density gradient centrifugation and cultured in DMEM $+10 \%$ fetal bovine serum until passage four. Morphological features, proliferation, cell surface marker expression and trilineage differentiation potential were assessed for both PB-MSCs and BM-MSCs. No significant differences in morphological features were observed. BM-MSCs had a higher proliferative capability than PB-MSCs as measured by XTT assays. Both PB-MSCs and BM-MSCs had broadly similar cell surface marker expression, but PB-MSCs had positive expression of cluster of differentiation (CD)146 and CD140b. Both PB-MSCs and BM-MSCs were capable of trilineage differentiation. Although BM-MSCs had a greater capacity for osteogenic
\end{abstract}

Correspondence to: Dr Ahmed Lotfy, Biotechnology and Life Sciences Department, Faculty of Postgraduate Studies for Advanced Sciences, Beni-Suef University, 1 Salah Salem Street, Beni-Suef 62511, Egypt

E-mail: lotfy_bio@hotmail.com

Key words: mesenchymal stem cells, peripheral blood, bone marrow, bone marrow-mesenchymal stromal cells, peripheral blood-mesenchymal stromal cells, BALB/c mice and chondrogenic differentiation than PB-MSCs, PB-MSCs had a better capability for adipogenic differentiation than BM-MSCs. In conclusion, PB-MSCs and BM-MSCs have very similar biological characteristics. Thus, $\mathrm{PB}$ is a promising source for easily obtaining MSCs in mice.

\section{Introduction}

Mesenchymal stem cells (MSCs) are adult stem cells that have a promising role in regenerative medicine (1). MSCs are multipotent and self-renewing cells and can differentiate into several types of cells (2). These cells are suitable for use in clinical applications because of their various properties, such as low immunogenicity, immunomodulatory effects (3), migration potential to sites of injury (4) and regenerative potential (5). MSCs can be isolated from different sources, such as bone marrow (6), adipose tissue (7), umbilical cord (8), and dental pulp (9).

MSCs were first isolated from bone marrow (10). Then, research focused on the identification of a less invasive source of MSCs than bone marrow. Harvesting adipose-derived stem cells (ASCs) is less invasive than harvesting bone marrow stem cells (BM-SCs) and can be performed during liposuction and preferably in cases of autologous therapy $(11,12)$. However, ASCs face further obstacles in the isolation steps, as they require digestion of adipose tissue using collagenase (7), which may affect cell viability. Therefore, identification of an alternative noninvasive source that is suitable for autologous therapy and requires minimal manipulation is needed.

Recently, MSCs have been successfully isolated from the peripheral blood of rats, rabbits, canines, ovines and equines (13-19). The major problem with this source is the low levels of stem cells among mononuclear cells. Researchers tried to increase the number of stem cells in peripheral blood by injection of mobilizing agents, such granulocyte colony-stimulating factor (20). The present study 
hypothesized that MSCs are found in peripheral blood at a certain percentage in mice and this percentage is inversely proportionate to age; its peak should be in the first weeks after birth.

In this study peripheral blood MSCs (PB-MSCs), were isolated from 4-week-old BALB/c white mice without using prior mobilizing agents and compared them with bone marrow MSCs (BM-MSCs).

\section{Materials and methods}

Ethical approval. The experimental protocol was approved by the Local Ethical Committee of the Faculty of Medicine, Mansoura University (Mansoura, Egypt) R/16.12.24.

Isolation of MSCs from BM and PB. MSCs were isolated from 4-week-old male BALB/c mice (weight, 15-20 g). A total of 6 mice were provided by Medical Experimental research Centre at the Faculty of Medicine, Mansoura University. Animals were housed in plastic cages (3/cage) on sawdust, with free access to food and water and were kept at a constant temperature of $22 \pm 1^{\circ} \mathrm{C}$ with $50 \%$ relative humidity and 12-h light/dark cycles for $\geq 1$ week before the experiment. $\mathrm{BM}$ was isolated from mouse femurs and tibiae as previously described (21). PB was isolated from the same mouse by cardiac puncture as previously described (22). In both samples, mononuclear cells were isolated by density gradient centrifugation $\left(400 \mathrm{x} \mathrm{g} ; 30 \mathrm{~min} ; 20^{\circ} \mathrm{C}\right)$ and cultured in DMEM supplemented with $10 \%$ FBS and $1 \%$ antibiotic-antimycotic solution (all Thermo Fisher Scientific, Inc.) in $25 \mathrm{~cm}^{2}$ flasks. Flasks were incubated at $37^{\circ} \mathrm{C}$ with $5 \% \mathrm{CO}_{2}$ and the cells were cultured until passage 4 .

XTT cell proliferation assay. In the XTT assay, passage 4 MSC proliferation was indirectly assessed by measuring metabolically active cells (23). MSCs were seeded at 125, 250, 500 and 1,000 cells/well and allowed to grow for 7 days at different concentrations. Optical density was measured at $450 \mathrm{~nm}$ after the addition of the XTT reagent (Roche Diagnostics). The assay was repeated three times.

Flow cytometric analysis. Passage 4 MSCs were characterized using cell surface markers by fluorescence-activated cell sorting analyses. The cells were stained with fluorescently labelled monoclonal antibodies against CD29, CD44, CD105, CD90.2, CD146, Sca-1, CD45 and CD140b (1 $\mu \mathrm{g} / \mathrm{ml}$; Miltenyi Biotec, Inc.; Table I) for $10 \mathrm{~min}$ in the dark at $2-8^{\circ} \mathrm{C}$. All data were acquired using a flow cytometer and assessed using FACSDiva v8.0.1 (BD Biosciences).

\section{Differentiation capability}

Osteogenic differentiation. Passage 4 MSCs were harvested, counted and seeded at a density of $5 \times 10^{4}$ per well in a 6 -well plate in osteogenesis differentiation media (DMEM supplemented with $10 \%$ FBS, $0.1 \mu \mathrm{M}$ dexamethasone, $100 \mu \mathrm{M}$ ascorbic acid and $10 \mathrm{mM} \beta$-glycerol phosphate from Sigma-Aldrich, Merck KGaA). The medium was changed twice per week for 2-3 weeks. The differentiation potential for osteogenesis was assessed by $40 \mathrm{mM}$ Alizarin Red ( $\mathrm{pH} 4.1)$ for $15 \mathrm{~min}$ at room temperature after fixation in $10 \%$ neutral buffered formalin for $10 \mathrm{~min}$ at room temperature. Quantification of calcium deposition was performed with a commercial calcium assay kit (Sigma-Aldrich, Merck KGaA). The assay was repeated three times.

Adipogenic differentiation. Passage 4 MSCs were harvested, counted and seeded at a density of $5 \times 10^{4}$ per well in a 24-well plate in adipogenesis differentiation media (DMEM supplemented with $10 \%$ FBS, $10 \%$ HS, $0.5 \mathrm{mM}$ isobutylmethylxanthine, $60 \mathrm{mM}$ indomethacin and $0.5 \mathrm{mM}$ hydrocortisone from Sigma-Aldrich, Merck KGaA); the medium was changed twice per week for 2 weeks. The differentiation potential for adipogenesis and formation of intracellular lipid droplets were assessed by Oil red $\mathrm{O}$ for $15 \mathrm{~min}$ at room temperature after fixation in $10 \%$ neutral buffered formalin for $10 \mathrm{~min}$ at room temperature using semi quantitative scoring as described by Aldridge et al (24). The level of adipogenesis was evaluated by ranking 500 cells in the wells by their fat content. Ranks were divided on the basis of the fat proportion: Grade 1, 0-24\%; grade 2, 25-49\%; grade 3, 50-74\%; grade 4, 75-100\%. The assay was repeated three times.

Chondrogenic differentiation. Passage 4 MSCs were harvested, counted and seeded at a density of $0.25 \times 10^{6}$ per Eppendorf tube in chondrogenic differentiation media [high-glucose DMEM supplemented with $10 \mathrm{ng} / \mathrm{ml}$ TGF- $\beta 3$, $100 \mathrm{nM}$ dexamethasone, $200 \mu \mathrm{M}$ ascorbate-2-phosphate, $40 \mu \mathrm{g} / \mathrm{ml}$ proline, $1 \mathrm{mM}$ pyruvate, $1 \mathrm{mg} / \mathrm{ml}$ bovine serum albumin (Sigma-Aldrich, Merck $\mathrm{KGaA}$ ) and $50 \mathrm{mg} / \mathrm{ml}$ ITS +3]. The medium was replaced every 2-3 days for 21 days. Cell pellets were fixed in $10 \%$ formalin for 1 day at room temperature and embedded in paraffin wax at $58^{\circ} \mathrm{C}$ for $15 \mathrm{~min}$. Sections of the cell pellets $(5 \mu \mathrm{m})$ were stained with toluidine blue for $30 \mathrm{~min}$ at $37^{\circ} \mathrm{C}(1 \%$ in $50 \%$ isopropanol) to demonstrate collagen content and sulfated proteoglycans within the extracellular matrix, indicated by blue color. Furthermore the production of sulfated GAG was measured in an Alcian blue binding assay (cat. no. 74240; Immunodiagnostic Systems) following digestion in $100 \mu 1$ papain solution. Absorbance was read at $630 \mathrm{~nm}$. The assay was repeated three times.

Statistical analysis. Data were presented as the mean \pm standard deviation. Statistical differences between groups were analyzed by one-way analysis of variance followed by Tukey's post hoc test with a Stata 7.0 software package (StataCorp LLC.). All assays were repeated three times. $\mathrm{P}<0.05$ was considered to indicate a statistically significant difference.

\section{Results}

XTT assay. BM-MSCs showed a significantly greater optical density in XTT assays compared with PB-MSCs $(\mathrm{P}<0.05)$ and the difference increased as the cell number increased, indicating that BM-MSCs have a higher proliferative rate than PB-MSCs (Fig. 1).

Flow cytometric analysis. Cultures of passage 4 BM-MSCs and PB-MSCs were analyzed for the expression of cell-surface markers (Fig. 2). BM-MSCs were positive for CD29 and 
Table I. Flow cytometry antibody panel used for characterization.

\begin{tabular}{lllll}
\hline Name & Conjugate & Clone & Cat. no. & Supplier \\
\hline CD29 & PE & HMs1-1 & $130-102-994$ & Miltenyi Biotec, Inc. \\
CD44 & FITC & IM7.8.1 & $130-102-511$ & Miltenyi Biotec, Inc. \\
CD105 & PE & MJ7/18 & $130-102-548$ & Miltenyi Biotec, Inc. \\
CD90.2 & FITC & $30-H 12$ & $130-120-091$ & Miltenyi Biotec, Inc. \\
CD146 & FITC & ME-9F1 & $130-102-230$ & Miltenyi Biotec, Inc. \\
SCa-1 & PE & D7 & $130-102-832$ & Miltenyi Biotec, Inc. \\
CD45 & FITC & $30 F 11.1$ & $130-110-658$ & Miltenyi Biotec, Inc. \\
CD140b & PE & APB5 & $130-118-457$ & Miltenyi Biotec, Inc.
\end{tabular}

$\mathrm{CD}$, cluster of differentiation; PE, phycoethrin; FITC, fluorescein isothiocyanate.

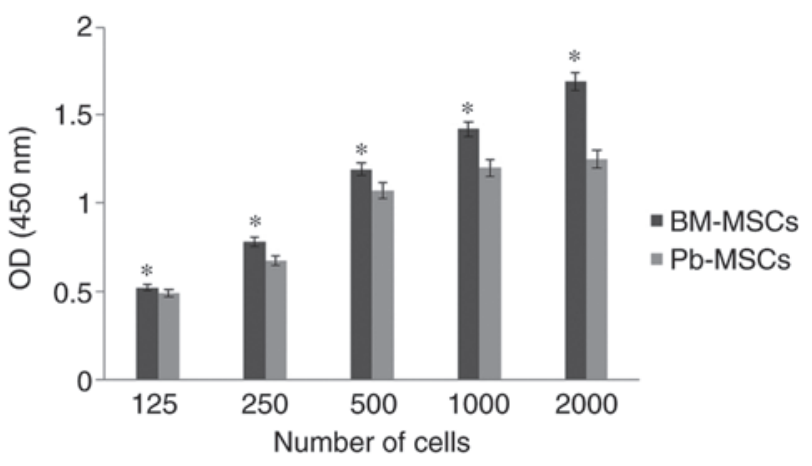

Figure 1. XTT assays of BM-MSCs and PB-MSCs to measure the proliferation rate. Both MSCs were seeded at 125, 250, 500 and 1,000 cells/well and allowed to grow for 7 days then the optical density was measured. The proliferation rate of BM-MSCs was higher than that of PB-MSCs and it was directly proportional to the number of inoculated cells. ${ }^{*} \mathrm{P}<0.05$ vs. $\mathrm{PB}-\mathrm{MSCs}$ BM-MSCs, bone marrow-mesenchymal stromal cells; PB, peripheral blood; OD, optical density.

negative for all other markers. PB-MSCs were positive for CD146, CD29 and CD140b and negative for Sca-1, CD44, CD45, CD90 and CD105.

\section{Differentiation capability}

Osteogenic differentiation. BM-MSCs and PB-MSCs differentiated into osteoblasts (Fig. 3A and B). BM-MSCs appeared to have a greater capability to differentiate into osteoblasts than PB-MSCs. Quantification of calcium deposition showed $2.12 \pm 0.106 \mu \mathrm{g} / \mathrm{ml}$ differentiated BM-MSC osteoblasts and $1.91 \pm 0.6 \mu \mathrm{g} / \mathrm{ml}$ differentiated PB-MSC osteoblasts (Fig. 4A).

Adipogenic differentiation. BM-MSC and PB-MSC differentiated into adipocyte (Fig. 3C and D) and PB-MSC exhibited a higher propensity to differentiate into adipocytes (Fig. 4B).

Chondrogenic differentiation. BM-MSCs and PB-MSCs tended to differentiate into chondrocytes (Fig. 3E and F), but BM-MSCs showed stronger production of sulfated GAG compared with PB-MSCs. The concentration of sulfated GAG in differentiated BM-MSCs was $17.93 \pm 2.44 \mu \mathrm{g} / \mathrm{ml}$. The concentration of sulfated GAG in differentiated PB-MSCs was $9.66 \pm 1.02 \mu \mathrm{g} / \mathrm{ml}$ (Fig. 4C).

\section{Discussion}

Bone marrow is the first and most common source of MSCs, but because collection of bone marrow is highly invasive, scientists have tried to find an alternative source, such as adipose tissue. Although adipose tissue is a less invasive source than bone marrow, there are still problems in its processing and digestion. PB is also an easy source of MSCs. In this study, the biological characteristics of mouse PB-MSCs and BM-MSCs were compared with regard to the proliferation rate, surface markers and trilineage differentiation potential (osteogenic, adipogenic and chondrogenic).

The XTT assay showed that the proliferation rate of BM-MSCs was compared with the PB-MSCs when cells were cultured in a gradual concentration and were left for a week, which is consistent with Fu et al (16), who compared BM-MSCs and PB-MSCs in rats. Immunophenotypic characterization also showed some differences, including CD146- and CD140b-positive expression only in PB-MSCs. CD146 expression in the MSC population showed heterogeneity in general and Espagnolle et al (25) demonstrated that MSCs with low CD146 expression had higher proliferation rates than MSCs with high CD146, which is consistent with the present study because PB-MSCs have high CD146 and low proliferation rates compared with $\mathrm{BM}-\mathrm{MSCs}$.

Both PB-MSCs and BM-MSCs could differentiate into osteoblasts, adipocytes and chondrocytes after these cells were cultured in differentiation-induction media compared with the control media. BM-MSCs showed higher differentiation potential to osteoblasts and chondrocytes than PB-MSCs based on their calcium and GAG accumulation, which is consistent with the findings of Lyahyai et al (26) and Spaas et al (27). In contrast to Chong et al (28), PB-MSCs showed higher adipogenic differentiation than BM-MSCs as assessed by fat droplet formation. MSC circulation in the bloodstream has been reported, but the exact tissue origin is debated. One theory states that PB-MSCs migrate from bone marrow, but the present study does not support this hypothesis because there are some biological differences $(13,29)$. 

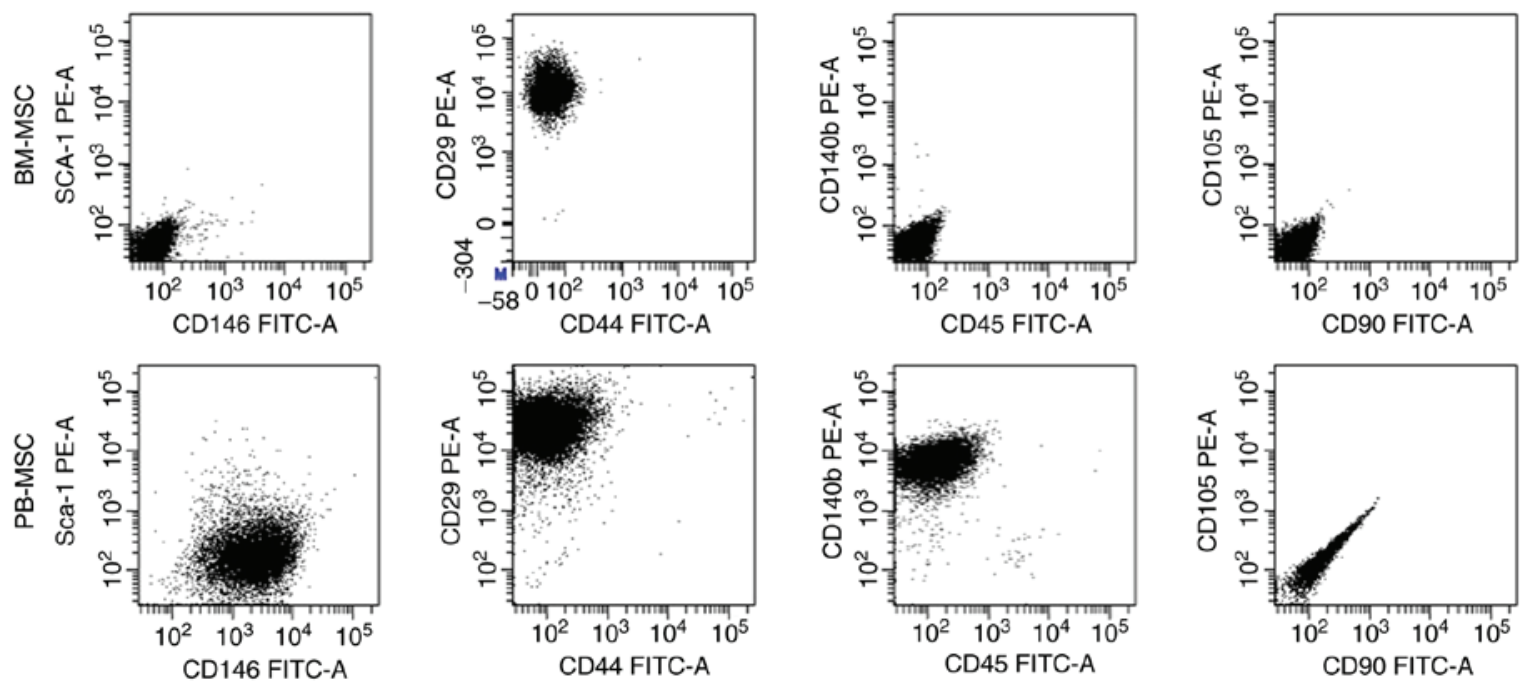

Figure 2. Flow cytometric analysis of BM-MSCs and PB-MSCs. BM-MSCs were positive CD29 and negative for other markers, while PB-MSCs were positive for CD146, CD29, and CD140b and negative for Sca-1, CD44, CD45, CD90 and CD105. CD, cluster of differentiation; BM-MSCs, bone marrow-mesenchymal stromal cells; PB, peripheral blood; FITC, fluorescein isothiocyanate; PE, phycoerythrin.
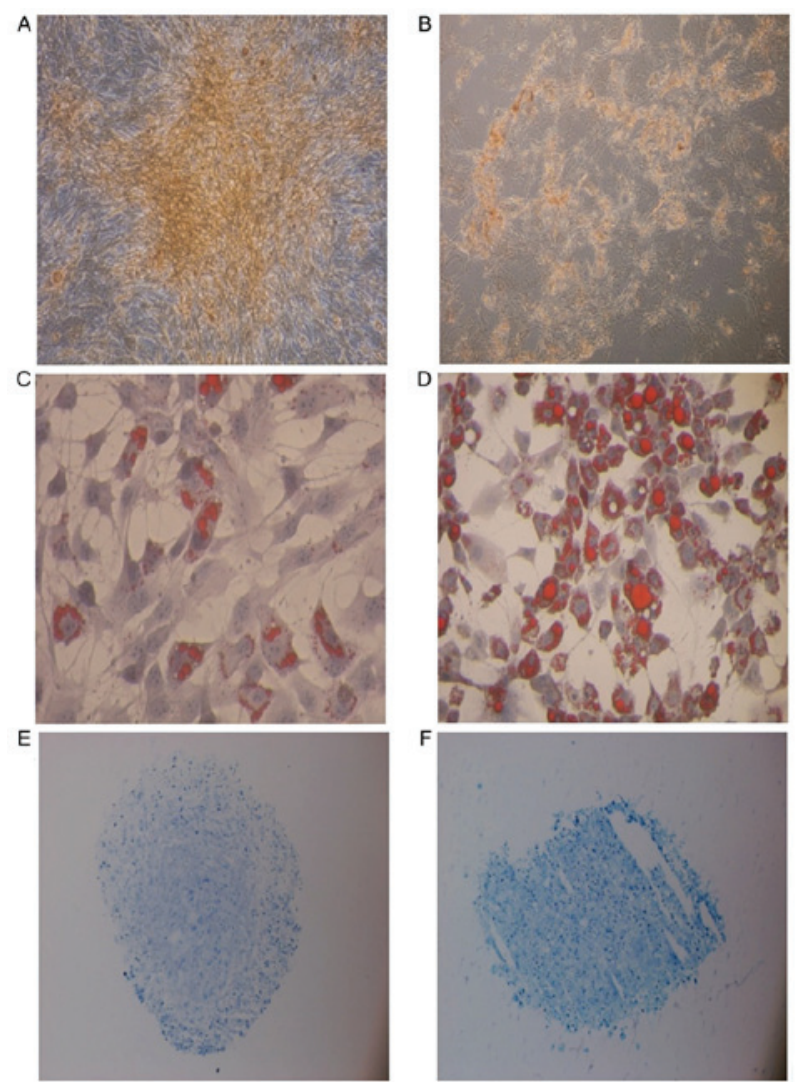

Figure 3. Differentiation capability of BM-MSCs and PB-MSCs. Osteogenic differentiation of (A) BM-MSCs and (B) PB-MSCs. Both stained with Alizarin Red (magnification, x10). Adipogenic differentiation of (C) BM-MSCs and (D) PB-MSCs stained with Oil red O (magnification, x10). Chondrogenic differentiation of (E) BM-MSCs and (F) PB-MSCs stained with toluidine blue (magnification, $\mathrm{x} 4$ ). BM-MSCs, bone marrow-mesenchymal stem cells; $\mathrm{PB}$, peripheral blood.

PB-MSCs are promising for autologous regenerative therapy. Blood samples taken from healthy children could be a source of stem cell banks for both autologous and off-shelf allogeneic therapy.
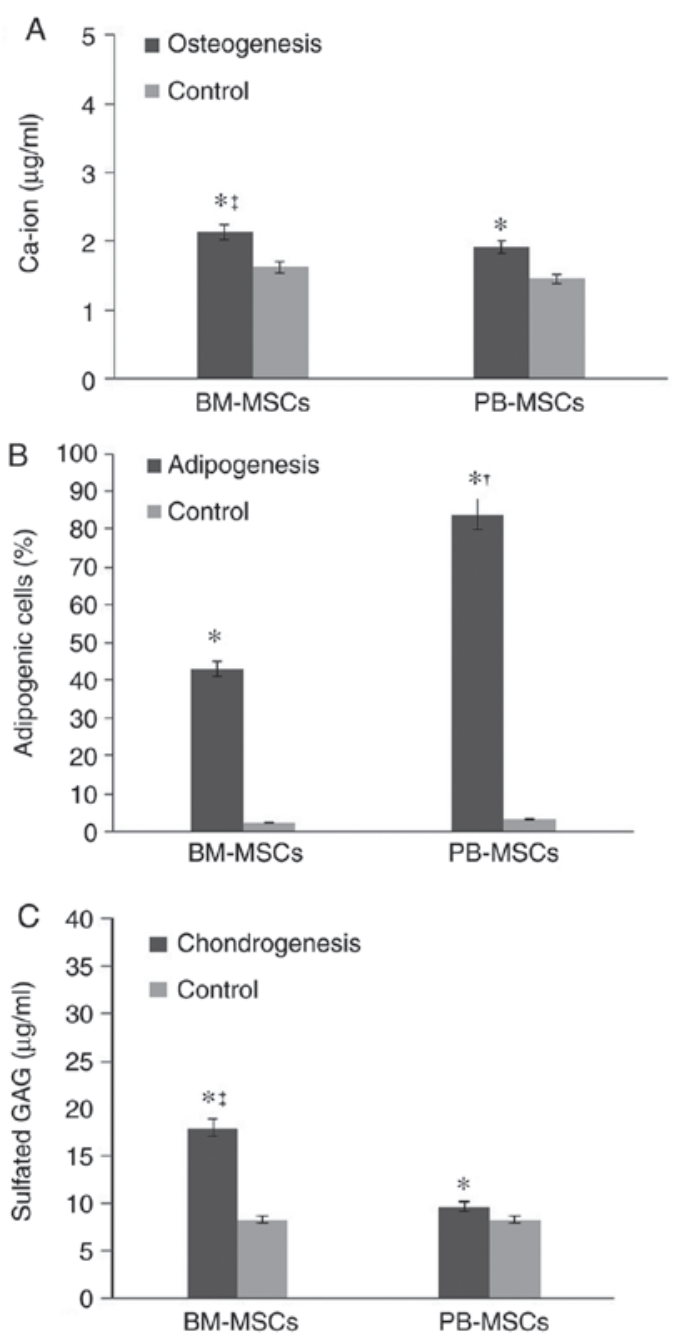

Figure 4. Quantitative analysis of the differentiation capability of BM-MSCs and PB-MSCs. (A) Osteogenesis detection by Ca-ion assays. (B) The adipogenesis percentage in PB-MSCs and BM-MSCs. (C) Chondro detection by GAG assay. Data are shown as the mean \pm standard deviation $(\mathrm{n}=3)$. ${ }^{*} \mathrm{P}<0.05$ vs. the control, ${ }^{\dagger} \mathrm{P}<0.05$ vs. BM-MSCs, ${ }^{\dagger} \mathrm{P}<0.05$ vs. PB-MSCs. BM-MSCs, bone marrow-mesenchymal stromal cells; PB, peripheral blood; chondro, chondrogenesis. 
In conclusion, $\mathrm{PB}-\mathrm{MSC}$ are easily obtained from the $\mathrm{PB}$ of young mice. Although PB-MSCs and BM-MSCs have some differences in differentiation and surface markers, they have very similar biological characteristics. Mouse PB-MSCs are a good source of MSCs and a parallel study of PB-MSCs and BM-MSCs in mice can shed more light on their biology in relation to ageing.

\section{Acknowledgements}

The authors would like to thank Professor Mohamed Sobh (Mansoura University) and Dr Jehan EL-Jawhari (University of Leeds) for their valuable support and advise throughout the characterization steps of the current study.

\section{Funding}

The present study was supported by the Science and Technology Development Fund (grant no. 4223).

\section{Availability of data and materials}

The data used to support the findings of this study are available from the corresponding author upon request.

\section{Authors' contributions}

AL, YMES, EJ and AB contributed to the study design. AL, YMES and RC contributed data acquisition and analysis. All authors contributed to writing and revising the manuscript.

\section{Ethics approval and consent to participate}

The experimental protocol was approved by the Local Ethical Committee of the Faculty of Medicine, Mansoura University (Mansoura, Egypt; no. R/16.12.24).

\section{Patient consent for publication}

Not applicable.

\section{Competing interests}

The authors declare that they have no competing interests.

\section{References}

1. Si YL, Zhao YL, Hao HJ, Fu XB and Han WD: MSCs: Biological characteristics, clinical applications and their outstanding concerns. Ageing Res Rev 10: 93-103, 2011.

2. Vater C, Kasten P and Stiehler M: Culture media for the differentiation of mesenchymal stromal cells. Acta Biomater 7: 463-477, 2011.

3. Yagi H, Soto-Gutierrez A, Parekkadan B, Kitagawa Y, Tompkins RG, Kobayashi N and Yarmush ML: Mesenchymal stem cells: Mechanisms of immunomodulation and homing. Cell Transplant 19: 667-679, 2010.

4. Sohni A and Verfaillie CM: Mesenchymal stem cells migration homing and tracking. Stem Cells Int 2013: 130763, 2013.

5. Bianchi F, Sala E, Donadei C, Capelli I and La Manna G: Potential advantages of acute kidney injury management by mesenchymal stem cells. World J Stem Cells 6: 644-650, 2014.
6. Friedenstein AJ, Chailakhyan RK, Latsinik NV, Panasyuk AF and Keiliss-Borok IV: Stromal cells responsible for transferring the microenvironment of the hemopoietic tissues. Cloning in vitro and retransplantation in vivo. Transplantation 17: 331-340, 1974.

7. Zuk PA, Zhu M, Ashjian P, De Ugarte DA, Huang JI, Mizuno H, Alfonso ZC, Fraser JK, Benhaim P and Hedrick MH: Human adipose tissue is a source of multipotent stem cells. Mol Biol Cell 13: 4279-4295, 2002.

8. Wang HS, Hung SC, Peng ST, Huang CC, Wei HM, Guo YJ, Fu YS, Lai MC and Chen CC: Mesenchymal stem cells in the Wharton's jelly of the human umbilical cord. Stem Cells 22: 1330-1337, 2004.

9. Liu J, Yu F, Sun Y, Jiang B, Zhang W, Yang J, Xu GT, Liang A and Liu S: Concise reviews: Characteristics and potential applications of human dental tissue-derived mesenchymal stem cells. Stem Cells 33: 627-638, 2015.

10. Friedenstein AJ, Chailakhjan RK and Lalykina KS: The development of fibroblast colonies in monolayer cultures of guinea-pig bone marrow and spleen cells. Cell Tissue Kinet 3: 393-403, 1970.

11. Harasymiak-Krzyzanowska I, Niedojadlo A, Karwat J, Kotula L, Gil-Kulik P, Sawiuk M and Kocki J: Adipose tissue-derived stem cells show considerable promise for regenerative medicine applications. Cell Mol Biol Lett 18: 479-493, 2013.

12. Lotfy A, Salama M, Zahran F, Jones E, Badawy A and Sobh M: Characterization of mesenchymal stem cells derived from rat bone marrow and adipose tissue: A comparative study. Int J Stem Cells 7: 135-142, 2014

13. He Q, Wan C and Li G: Concise review: Multipotent mesenchymal stromal cells in blood. Stem Cells 25: 69-77, 2007.

14. Koerner J, Nesic D, Romero JD, Brehm W, Mainil-Varlet P and Grogan SP: Equine peripheral blood-derived progenitors in comparison to bone marrow-derived mesenchymal stem cells. Stem Cells 24: 1613-1619, 2006.

15. Zvaifler NJ, Marinova-Mutafchieva L, Adams G, Edwards CJ, Moss J, Burger JA and Maini RN: Mesenchymal precursor cells in the blood of normal individuals. Arthritis Res 2: 477-488, 2000.

16. Fu WL, Zhang JY, Fu X, Duan XN, Leung KK, Jia ZQ, Wang WP, Zhou CY and Yu JK: Comparative study of the biological characteristics of mesenchymal stem cells from bone marrow and peripheral blood of rats. Tissue Eng Part A 18: 1793-1803, 2012.

17. Longhini ALF, Salazar TE, Vieira C, Trinh T, Duan Y, Pay LM, Li Calzi S, Losh M, Johnston NA, Xie H, et al: Peripheral blood-derived mesenchymal stem cells demonstrate immunomodulatory potential for therapeutic use in horses. PLoS One 14: e0212642, 2019.

18. Li S, Huang KJ, Wu JC, Hu MS, Sanyal M, Hu M, Longaker MT and Lorenz HP: Peripheral blood-derived mesenchymal stem cells: Candidate cells responsible for healing critical-sized calvarial bone defects. Stem Cells Transl Med 4: 359-368, 2015.

19. Fu Q, Tang NN, Zhang Q, Liu Y, Peng JC, Fang N, Yu LM, Liu JW and Zhang T: Preclinical study of cell therapy for osteonecrosis of the femoral head with allogenic peripheral blood-derived mesenchymal stem cells. Yonsei Med J 57: 1006-1015, 2016

20. Fruehauf S, Veldwijk MR, Seeger T, Schubert M, Laufs S, Topaly J, Wuchter P, Dillmann F, Eckstein V, Wenz F, et al: A combination of granulocyte-colony-stimulating factor (G-CSF) and plerixafor mobilizes more primitive peripheral blood progenitor cells than G-CSF alone: Results of a European phase II study. Cytotherapy 11: 992-1001, 2009.

21. Nadri S and Soleimani M: Isolation murine mesenchymal stem cells by positive selection. In Vitro Cell Dev Biol Anim 43: 276-282, 2007.

22. Kuznetsov SA, Mankani MH, Gronthos S, Satomura K, Bianco P and Robey PG: Circulating skeletal stem cells. J Cell Biol 153: 1133-1140, 2001.

23. Jones EA, English A, Henshaw K, Kinsey SE, Markham AF, Emery $\mathrm{P}$ and McGonagle D: Enumeration and phenotypic characterization of synovial fluid multipotential mesenchymal progenitor cells in inflammatory and degenerative arthritis. Arthritis Rheum 50: 817-827, 2004.

24. Aldridge A, Kouroupis D, Churchman S, English A, Ingham E and Jones E: Assay validation for the assessment of adipogenesis of multipotential stromal cells-a direct comparison of four different methods. Cytotherapy 15: 89-101, 2013. 
25. Espagnolle N, Guilloton F, Deschaseaux F, Gadelorge M Sensébé L and Bourin P: CD146 expression on mesenchymal stem cells is associated with their vascular smooth muscle commitment. J Cell Mol Med 18: 104-114, 2014.

26. Lyahyai J, Mediano DR, Ranera B, Sanz A, Remacha AR, Bolea R, Zaragoza P, Rodellar C and Martín-Burriel I: Isolation and characterization of ovine mesenchymal stem cells derived from peripheral blood. BMC Vet Res 8: 169, 2012.

27. Spaas JH, De Schauwer C, Cornillie P, Meyer E, Van Soom A and Van de Walle GR: Culture and characterisation of equine peripheral blood mesenchymal stromal cells. Vet J 195: 107-113, 2013.
28. Chong PP, Selvaratnam L, Abbas AA and Kamarul T: Human peripheral blood derived mesenchymal stem cells demonstrate similar characteristics and chondrogenic differentiation potential to bone marrow derived mesenchymal stem cells. J Orthop Res 30: 634-642, 2012.

29. Hass R, Kasper C, Böhm S and Jacobs R: Different populations and sources of human mesenchymal stem cells (MSC): A comparison of adult and neonatal tissue-derived MSC. Cell Commun Signal 9: 12, 2011. 